\section{Growing autologous bone}

$\mathrm{T}$ issue engineering has yet to yield a good source of bone for orthopedic surgery. So far, attempts to grow bone tissue in vitro have not been successful. ${ }^{1}$ However, new research by Molly Stevens and colleagues suggests that new bone can be engineered to grow in vivo and then used to repair damage elsewhere. ${ }^{1}$

Autologous bone is used in the treatment of severe fractures and bone tumour resections and for spinal fusions, of which more than 200000 are performed annually in the United States alone. ${ }^{1}$ Currently, living bone is often harvested from the iliac crest. However, this supply is limited, and harvesting results in pain and morbidity among patients. Tissue engineering is also a challenge since bone is a highly organized and complex tissue. Previous attempts at in vivo culture have involved using a mineralized bone scaffold placed in muscle tissue; ${ }^{2}$ the results of this procedure, however, $\stackrel{\sim}{\vec{\oplus}}$ have been unreliable.

Stevens and colleagues have

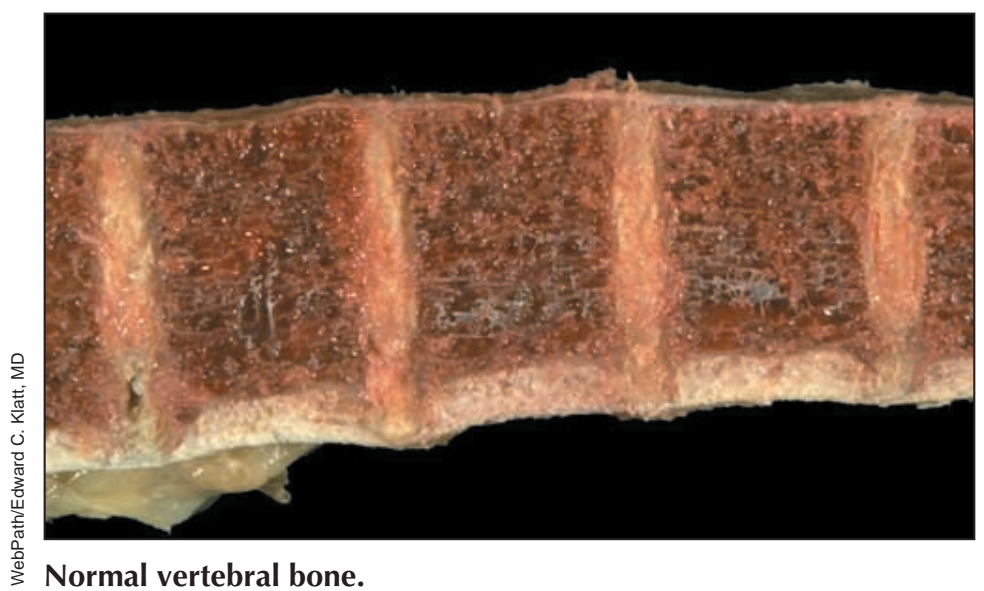

and in another 6 weeks it had become mature, compact bone.

It was unclear whether the new bone could be harvested and integrated into a recipient site. However, Stevens and colleagues found that the new bone had a demarcation that allowed it to be removed without compromising the surrounding bone. It was then grafted onto another site on the tibia, where it remodelled and integrated into the existing bone.

Whether an "in vivo bioreactor" will work in humans for the banking and transplantation of bone is still an open question. But Stevens and colleagues have already discovered that a $1-\mathrm{cm}$ space can be created between human tibiae and the mesenchymal cambium layer. ${ }^{1}$ This finding, combined with the ease of their method, which simply takes advantage of the natural healing process of the body, suggests that "bioreactors" may be an important new way to engineer a variety of tissues (Stevens and colleagues have also been able to create cartilage with the technique). ${ }^{1}$ As such, orthopedic surgeons may one day have an unlimited supply of bone grown right in the patients who need it. - David Secko, Vancouver

\section{References}

1. Stevens MM, Marini RP, Schaefer D Aronson J, Langer R, Shastri P. In vivo engineering of organs: the bone bioreactor. Proc Natl Acad Sci U S A 2005;102(32):11450-5.

2. Warnke PH, Springer IN, Wiltfang J, Acil Y, Eufinger H, Wehmoller M, et al. Growth and transplantation of a custom vascularised bone graft in a man. Lancet 2004;364(9436):766-70. 